\title{
ON POWER-BOUNDED PRESPECTRAL OPERATORS
}

\author{
by H. R. DOWSON \\ (Received 7th February 1975)
}

Foguel (8) and Fixman (7) independently proved that an invertible spectral operator, which is power-bounded, is of scalar type. Their proofs rely heavily on a result of Dunford on spectral operators whose resolvents satisfy a growth condition. (See Lemma 3.16 of (6, p. 609).) Observe that the resolvent of an invertible power-bounded operator $T$ satisfies an inequality of the form

$$
\left\|(\lambda I-T)^{-1}\right\| \leqq \frac{M}{|1-| \lambda||}(|\lambda| \neq 1) .
$$

This means that near the unit circle the resolvent has first-order rate of growth along radial lines. The main purpose of the present paper is to give a nicer proof of the theorem of Foguel and Fixman using the recently developed theory of hermitian operators on Banach spaces. In fact, our proof yields the more general result that an invertible prespectral operator, which is powerbounded, is a scalar-type operator.

It will be assumed that the reader is familiar with the definition and properties of prespectral operators given in (2) and (4). Throughout $X$ denotes a complex Banach space with dual space $X^{*}$. The Banach algebra of bounded linear operators on $X$ is denoted by $L(X)$. Let $T \in L(X)$. The spectrum of $T$ is denoted by $\sigma(T) . \quad Z$ denotes the set of integers and $R$ the set of real numbers.

Theorem 1. Let $T$, in $L(X)$, be an invertible prespectral operator of class $\Gamma$. Suppose that $T$ is power-bounded in the sense that there is a real number $K$ such that

$$
\left\|T^{n}\right\| \leqq K \quad(n \in Z) .
$$

Then $T$ is a scalar-type operator of class $\Gamma$.

Proof. Observe that

$$
\begin{gathered}
(\lambda I-T)^{-1}=\sum_{n=0}^{\infty} \frac{T^{n}}{\lambda^{n+1}} \quad(|\lambda|>1) \\
(\lambda I-T)^{-1}=-\sum_{n=0}^{\infty} \lambda^{n}\left(T^{-1}\right)^{n+1} \quad(|\lambda|<1)
\end{gathered}
$$

both series converging in the norm of $L(X)$. Hence $\sigma(T) \subseteq\{z:|z|=1\}$. By Theorem 1 of $(5 ;$ pp. 57-58) there is $H$, in $L(X)$, such that $\exp i H=T$ and $H$ is prespectral of class $\Gamma$. By the spectral mapping theorem $\sigma(H)$ is 
real. Consider the group of operators $\{\exp i \alpha H: \alpha \in R\}$. Since the map $\beta \rightarrow \exp i \beta H$ is a continuous map of $[0,1]$ into $L(X)$

Define

$$
\|\exp i \alpha H\| \leqq K \sup _{\beta \in[0,1]}\|\exp i \beta H\|<\infty \quad(\alpha \in \boldsymbol{R}) .
$$

$$
\|\| x \|=\sup \{\|\exp i \alpha H x\|: \alpha \in R\} \quad(x \in X) .
$$

Then \|\|$\|$ is a norm on $X$ equivalent to \|\| ; moreover

$$
\|\mid \exp i \alpha H\|=1 \quad(\alpha \in R) \text {. }
$$

This means that under an equivalent renorming of $X, H$ is hermitian. Let $E(\cdot)$ be the resolution of the identity of class $\Gamma$ for $H$. Define

$$
\begin{aligned}
& R=\int_{\sigma(H)} \lambda E(d \lambda)=\int_{\sigma(H)} \operatorname{Re} \lambda E(d \lambda), \\
& Q=H+R .
\end{aligned}
$$

Then by Theorem 2.5 of $(1 ;$ p. 368) there is an equivalent norm on $X$ with respect to which $R$ is hermitian. Since $H R=R H$ then by Corollary 7 of $(9 ;$ p. 78) there is an equivalent norm on $X$ with respect to which $H$ and $R$ are simultaneously hermitian. Assume that this renorming has been carried out. Then in the equation $H-R=Q$, the left-hand side is hermitian and the right-hand side quasinilpotent. Hence by Sinclair's theorem $(3 ;$ p. 73) $Q=0$ and $H=R$. Thus $H$ is a scalar-type operator of class $\Gamma$ and by Theorem 3.1 of $(2 ;$ p. 294) so is $T=\exp i H$.

Theorem 2. Let $T$, in $L(X)$, be an invertible spectral operator which is powerbounded in the sense that there is a real number $K$ such that

$$
\left\|T^{n}\right\| \leqq K \quad(n \in Z) .
$$

Then $T$ is a scalar-type spectral operator.

This result, first proved by Foguel (Theorem 3 of $(8 ;$ p. 62)) and Fixman (Theorem 4.2 of $\left(7 ;\right.$ p. 104)), follows from Theorem 1 on taking $\Gamma=X^{*}$.

\section{REFERENCES}

(1) E. Berkson, A characterization of scalar-type operators on reflexive Banach spaces, Pacific J. Math. 13 (1963), 365-373.

(2) E. Berkson and H. R. Dowson, Prespectral operators, Illinois J. Math. 13 (1969), 291-315.

(3) F. F. Bonsall and J. Duncan, Numerical Ranges II (London Math. Soc. Lecture Note Series No. 10, 1973).

(4) H. R. Dowson, A commutativity theorem for prespectral operators, Illinois J. Math. 17 (1973), 525-532.

(5) H. R. Dowson, Logarithms of prespectral operators, J. London Math. Soc. (2) 9 (1974), 57-64. 
(6) N. Dunford, Spectral theory II. Resolutions of the identity, Pacific J. Math. 2 (1952), 559-614.

(7) U. Fixman, Problems in spectral operators, Pacific J. Math. 9 (1959), 1029 1051.

(8) S. R. FogueL, The relations between a spectral operator and its scalar part, Pacific J. Math. 8 (1958), 51-65.

(9) G. Lumer, Spectral operators, hermitian operators and bounded groups, Acta Sci. Math. (Szeged) 25 (1964), 75-85.

UNIVERSITY OF GLASGOW

GLASGOW G12 8QW 